\title{
Depressive symptoms, physical inactivity and risk of cardiovascular mortality in older adults: the Cardiovascular Health Study
}

\author{
Sithu Win, ${ }^{1}$ Kapil Parakh, ${ }^{1}$ Chete M Eze-Nliam, ${ }^{2}$ John S Gottdiener, ${ }^{3}$ Willem J Kop, ${ }^{3}$ \\ Roy C Ziegelstein ${ }^{1}$
}

'Department of Medicine, Johns Hopkins University School of Medicine, Baltimore, Maryland, USA

${ }^{2}$ Department of Psychiatry and Behavioural Sciences, Johns Hopkins University School of Medicine, Baltimore, Maryland USA

${ }^{3}$ Department of Medicine, University of Maryland School of Medicine, Baltimore, Maryland, USA

\section{Correspondence to}

Roy C Ziegelstein, Johns Hopkins University School of Medicine, Department of Medicine, B-1-North, Johns Hopkins Bayview Medical Center, 4940 Eastern Avenue, Baltimore, MD 21224-2780, USA; rziegel2@jhmi.edu

Accepted 23 December 2010

\begin{abstract}
Background Depressed older individuals have a higher mortality than older persons without depression. Depression is associated with physical inactivity, and low levels of physical activity have been shown in some cohorts to be a partial mediator of the relationship between depression and cardiovascular events and mortality.

Methods A cohort of 5888 individuals (mean $72.8 \pm 5.6$ years, $58 \%$ female, $16 \%$ African-American) from four US communities was followed for an average of 10.3 years. Self-reported depressive symptoms (10-item Center for Epidemiological Studies Depression Scale) were assessed annually and self-reported physical activity was assessed at baseline and at 3 and 7 years. To estimate how much of the increased risk of cardiovascular mortality associated with depressive symptoms was due to physical inactivity, Cox regression with time-varying covariates was used to determine the percentage change in the log HR of depressive symptoms for cardiovascular mortality after adding physical activity variables.
\end{abstract}

Results At baseline, 20\% of participants scored above the cut-off for depressive symptoms. There were 2915 deaths (49.8\%), of which 1176 (20.1\%) were from cardiovascular causes. Depressive symptoms and physical inactivity each independently increased the risk of cardiovascular mortality and were strongly associated with each other (all $p<0.001$ ). Individuals with both depressive symptoms and physical inactivity had greater cardiovascular mortality than those with either individually $(p<0.001, \log$ rank test). Physical inactivity reduced the log HR of depressive symptoms for cardiovascular mortality by $26 \%$ after adjustment. This was similar for persons with (25\%) and without (23\%) established coronary heart disease.

Conclusions Physical inactivity accounted for a significant proportion of the risk of cardiovascular mortality due to depressive symptoms in older adults, regardless of coronary heart disease status.

\section{INTRODUCTION}

Depression has been associated with increased cardiovascular mortality in older individuals in most, ${ }^{1-4}$ but not all, ${ }^{5}{ }^{6}$ studies. Multiple potential behavioural and biological mechanisms have been proposed to explain this association, ${ }^{7}$ and it is likely that several mediating factors play a role. One important plausible mediating factor to consider is physical inactivity since many patients with depression are physically inactive. ${ }^{89}$ In addition, a strong inverse relationship exists between selfreported activity levels, ${ }^{10-16}$ as well as objective measures of daily energy expenditure, ${ }^{16}$ and mortality risk. The adverse health consequences of physical inactivity are greater in adults aged $\geq 65$ years than in younger individuals. ${ }^{10-13}$

Physical inactivity has been shown partially to mediate the relationship between depression and mortality among persons with established cardiovascular disease, ${ }^{7} 1718$ but the possibility that physical inactivity mediates the relationship between depression and mortality in individuals without known heart disease is not well documented using longitudinal assessments. In the Finland, Italy and the Netherlands Elderly (FINE) study, ${ }^{19}$ physical inactivity explained only a small percentage $(9 \%)$ of the increased mortality risk due to depressive symptoms in men aged 70-90 years who were free of cardiovascular disease at baseline.

The Cardiovascular Health Study (CHS) is a prospective observational study of communitydwelling individuals aged $\geq 65$ years from whom extensive repeated evaluations of health information have been collected by systematic interviews and clinical examination. Previous studies have shown that both physical inactivity ${ }^{12}$ and depressive symptoms ${ }^{4}$ independently predict increased mortality in this cohort of individuals. The present study examined whether physical inactivity is a mediator of the increased risk of cardiovascular mortality associated with depressive symptoms among community dwelling older adults, adjusting for demographic variables, comorbid medical conditions and health behaviours.

\section{METHODS}

\section{Study population}

The CHS is a multicentre prospective cohort study of cardiovascular risk factors in ambulatory noninstitutionalised men and women aged $\geq 65$ years Participants were randomly selected using Medicare eligibility lists of the Health Care Financing Administration from four communities in the USA: Washington County, Maryland; Forsyth County, North Carolina; Allegheny County, Pennsylvania; and Sacramento County, California. Initial recruitment of 5201 subjects occurred in 1989 with an additional 687 African-Americans recruited 3 years later, bringing the total to 5888 participants. The institutional review board at each centre approved the study and participants gave informed consent. online under the BMJ Journals unlocked scheme, see http:// heart.bmi.com/site/about/ unlocked.xhtml 
Additional details of the study design and recruitment process have been published previously. ${ }^{20} 21$

The baseline visit included a standardised physical examination and questionnaire, laboratory testing and diagnostic evaluation. Participants returned annually for nine additional clinic visits and were also contacted by telephone every 6 months. Individual follow-up was available up to 14 years, the date of last known contact or death, whichever occurred first.

Information was gathered on smoking status, alcohol consumption, medications and medical conditions including coronary heart disease (defined as angina, previous myocardial infarction or coronary revascularisation), heart failure, stroke, hypertension and diabetes. Examination included measurement of body mass index, waist-to-hip ratio and seated blood pressure using a random zero sphygmomanometer. Participants also had a standard resting 12-lead ECG, echocardiogram and laboratory tests including serum cholesterol, glucose, creatinine and $\mathrm{C}$ reactive protein.

\section{Assessment of physical activity}

Usual physical activity was assessed by a self-report questionnaire at baseline and again after 3 and 7 years. Four aspects of physical activity were evaluated:

1. Leisure time activity was assessed by a modified Minnesota Leisure-Time Activities questionnaire which asked participants about 15 different activities during the previous 2 weeks. $^{22}$ The questionnaire has been validated previously in other cohorts. ${ }^{23}$ Responses were used to estimate energy expenditure in kilocalories per week and then categorised into quintiles.

2. Exercise was categorised as no exercise, low, moderate and high intensity as previously described. ${ }^{24}$ Briefly, participants who engaged in at least one of swimming, hiking, aerobics, tennis, jogging or racquetball or who walked for exercise at a brisk ( $>4 \mathrm{mph}$ ) pace were categorised as having engaged in high-intensity activity; those who engaged in at least one of gardening, mowing, raking, golf, bowling, biking, dancing, calisthenics or exercise cycle or who walked for exercise at an average $(>2-3 \mathrm{mph}$ ) or fairly brisk pace ( $>3-4 \mathrm{mph}$ ) were categorised as having engaged in moderate intensity activity; and participants who did not report participating in any of the 15 leisure time activities or who walked for exercise at a casual or strolling pace ( $<2 \mathrm{mph}$ ) were categorised as having engaged in low intensity activity.

3. Distance walked in blocks was categorised into quintiles.

4. Pace of walking was categorised as no walking, $<2,2-3,3-4$ and $>4 \mathrm{mph}$.

To obtain an overall assessment of physical activity we created a composite Physical Activity Score (PAS) from these four components. A score of 0 was assigned to the lowest category in each area with 1 point for each increase in each domain of physical activity. This provided a range of 0 (least active) to 15 (most active). This score was then categorised into four groups: PAS $0-3,4-7,8-11$ and $12-15$. The internal consistency of this scale was good (Cronbach's $\alpha=0.78$ ).

\section{Assessment of depressive symptoms}

The short (10-item) version ${ }^{25}$ of the Center for Epidemiological Studies Depression (CES-D) scale ${ }^{26}$ was used annually to assess self-reported depressive symptoms experienced in the past week. This version of the CES-D has shown good validity versus the 20-item CES-D, particularly in epidemiological studies and older populations. ${ }^{25}$ The scale consists of 10 items, each scored $0-3$, for a maximum of 30 points. Higher scores indicate greater frequency of depressive symptoms and correlate with an increased risk of clinical depression.

Depression scores were dichotomised at each visit with a cutoff of $\geq 8$ as in previous studies in the CHS, ${ }^{4}{ }^{27}$ creating low (CES-D <8) and high (CES-D $\geq 8$ ) groups for analysis. The cut-off of 8 on the short version of the CES-D corresponds to a cut-off of $\geq 16$ on the 20 -item version of the CES-D.

\section{Assessment of events and cardiovascular mortality}

All events occurring after the baseline visit were classified as incident events and were adjudicated by a centralised committee. $^{22}$ The cause of death was determined from medical records, death certificates, ICD codes, obituaries and interviews with relatives and contacts. Cardiovascular deaths were those due to atherosclerotic coronary disease, cerebrovascular disease (stroke), other atherosclerotic disease (such as aortic aneurysm) and other vascular disease (such as valvular heart disease or pulmonary embolism). ${ }^{28}$ The CHS has nearly $100 \%$ ascertainment of mortality status.

\section{Statistical analysis}

Missing data for any visit the participant was known to have attended were replaced with data from other visits. Missing baseline data were filled with values from the next available visit. When data were missing at later visits, the last observation was carried forward. This was done to maintain a more consistent sample size, as it may vary substantially when using time-varying covariates in analyses. Approximately $5 \%$ of data was missing at any visit for any variable. Missing data on depressive symptoms ranged from $1.5 \%$ at baseline to $10 \%$ at later visits. Participants who had never had an assessment of physical activity or depressive symptoms at any time during the study were excluded $(n=36)$. Data from 5852 participants remained for analysis.

Baseline characteristics were compared between high and low depressive symptoms groups using t tests with unequal variance for continuous variables and $\chi^{2}$ tests for dichotomous variables. The characteristics of the physical activity groups were compared using univariate linear (continuous variables) and logistic (dichotomous variables) regression evaluating for trend. The association between physical activity and depressive symptoms at baseline was evaluated using multivariate linear and logistic regression models.

Cox proportional hazards regression was used to estimate the risk of cardiovascular mortality associated with depressive symptoms, physical inactivity and other covariates. In the models, participants were included up to the date of death or last known visit. The group of 687 African-Americans recruited 3 years after study initiation was treated as a late entry cohort and immortal person-time before recruitment was removed. Variables were chosen for the final model based on the significance of their univariate associations as well as clinical interest. The proportional hazards assumption was checked with a log-log plot of the survival function and was met for both depression and physical inactivity.

All variables were treated as time-varying. For example, the CES-D was administered at baseline and annually for 9 additional years, so individuals may have up to 10 measurements. After dichotomisation they may fall into both high and low depression score groups at different points during follow-up. The Cox models used all available measurements and alternated the person-time to the appropriate risk group. Using repeated measurements reduces misclassification bias and provides more accurate estimates of risk throughout follow-up and at the time 
of events. For incident events occurring between visits, that period was updated if it occurred in the first half. For example, if a participant suffered a stroke 4 months after his fifth annual visit, the period between the fifth and sixth visits and subsequent periods were reclassified as prevalent stroke. If it occurred at 8 months, only subsequent periods were reclassified.

To estimate how much of the increased risk of cardiovascular mortality due to depressive symptoms may be accounted for by physical inactivity, we determined the percentage change in the coefficient for depression after physical activity variables were individually added to Cox models. This was calculated as:

$\log \left(\mathrm{HR}_{\text {depression }}\right.$ [model without physical activity] $)-\log$ $\left(\mathrm{HR}_{\text {depression }}\right.$ [model with physical activity] $) / \log \left(\mathrm{HR}_{\text {depression }}\right.$ model without physical activity).

We considered this percentage change in the logHR of depression to be a measure of confounding or mediation. This was calculated for the PAS and for each of its components.

Subgroup analyses were performed in a similar fashion, stratifying by baseline coronary heart disease status, race and gender. We also performed sensitivity analyses by using only baseline data, no time-varying covariates and without filling in missing values. A two sided $p$ value $<0.05$ was considered statistically significant. All analyses were performed in STATA Version 10.1 (StataCorp LP).

\section{RESULTS}

At study entry the mean \pm SD age of the participants was $72.8 \pm 5.6$ years (range $65-100$ ); $58 \%$ were female and $16 \%$ were non-white, of whom $96 \%$ were African-Americans. Differences in characteristics of participants at study entry by depression score and physical activity group are shown in table 1. Participants with a CES-D score above the cut-off were more often female, non-white, current smokers, less educated, consumed less alcohol and had a greater prevalence of comorbidities. About $8 \%$ of those with high depression scores were taking antidepressants compared with $3 \%$ of the participants with low depression scores. Those who were more physically inactive were older, more often female, non-white, current smokers, less educated and consumed less alcohol than physically active participants. They had higher body mass index and blood pressure, more comorbidities and less favourable lipid and chemistry profiles than more physically active participants.

The percentage of participants with a CES-D score above the cut-off increased over time, ranging from $20 \%$ at baseline to $30 \%$ after 10 years. During this time the study population also became less physically active with an overall shift towards lower activity groups. The percentage of persons falling into the lowest activity group increased from 10\% at baseline to $19 \%$ at 10 years, while the percentage in the highest activity group fell from $14 \%$ to $9 \%$. Depression scores and physical activity levels were strongly associated. After adjustment for age, race and sex, persons in the lowest physical activity group were more likely to have a high depression score than those in the most active group (OR 3.4; 95\% CI 2.6 to $4.5 ; \mathrm{p}<0.001$ ). The results were similar when variables were treated as continuous and for each component of the PAS examined individually (not shown).

The mean follow-up duration was 10.3 years (maximum 14), which provided 60652 person-years of observation. Mean follow-up duration was shorter for non-white participants (9.1 years), in part because most (71\%) entered the study 3 years after the original cohort because of the recruitment of 687 African-Americans at that time. Overall, there were 2915 deaths (49.8\%), including 1176 cardiovascular deaths (20.1\%).
The risk for cardiovascular mortality stratified by level of depressive symptoms and by physical activity scores is shown in table 2. Persons with high depression scores had a $27 \%$ (multivariable adjusted) to $67 \%$ (unadjusted) increased risk of cardiovascular death compared with those with low depression scores. For physical activity, a stepwise increase in the risk of cardiovascular death was observed among progressively less active groups. Compared with those in the most active group, persons in the lowest activity group had a $217 \%$ (multivariable adjusted) to $425 \%$ (unadjusted) increased risk of cardiovascular mortality.

Table 3 shows the percentage reduction in the logHR of the depression score for cardiovascular mortality after adding physical activity to the multivariable adjusted models. In the full cohort the addition of PAS resulted in a $26 \%$ reduction in the $\operatorname{logHR}$ while its individual components accounted for reductions of $10-19 \%$. This effect was similar when the 4671 (79.8\%) participants without known coronary heart disease were compared with the 1181 (20.2\%) participants with known coronary heart disease.

Physical inactivity accounted for a greater percentage of the risk due to depression in men than in women. The addition of PAS reduced the logHR of depression by $31 \%$ in men and by $22 \%$ in women, with similar results in the individual components. PAS also tended to account for a greater percentage of the risk among white $(27 \%)$ compared with non-white $(11 \%)$ participants. However, the logHR of depression among non-whites in multivariable models did not achieve statistical significance (p range $0.16-0.24$ ).

In a Kaplan-Meier analysis (figure 1), physical inactivity (median PAS score $\leq 7$ ) combined with high depressive symptoms (CES-D $\geq 8$ ) were associated with a significantly greater risk than having either condition alone (both $p<0.001$, log rank test). At these cut-off values the risk due to physical inactivity was greater than the risk due to high depressive symptoms $(p=0.004, \log$ rank test). The magnitude of the increase in risk between inactive and active participants was the same in both depression groups ( $p$ for interaction $=0.251$, demographic adjusted), suggesting that the risks of depression and inactivity for cardiovascular death are additive. Overall, the results were similar when using only baseline data (without time-varying covariates) and without substitution of missing data.

\section{DISCUSSION}

This study shows that community-dwelling older adults in the CHS with high depression scores were at an increased risk of cardiovascular mortality during a follow-up period of approximately 10 years. Individuals with CES-D scores above the cut-off had a $67 \%$ greater risk of cardiovascular mortality than individuals with low depression scores, a risk that remained significant after adjustment for other important predictors of cardiovascular mortality. This finding alone is not surprising since previous reports from the CHS have shown that depression is associated with greater all-cause mortality ${ }^{4}$ and cardiovascular mortality $^{27}$ in this cohort. This study addressed the contribution of physical inactivity to the increased mortality risk related to depression, since physical inactivity has been associated with substantially higher mortality in the $\mathrm{CHS}^{12}$ and since depression is known to be associated with physical inactivity. $^{8} 9$ The major new finding of the present study is that physical inactivity accounts for approximately $25 \%$ of the increased risk of cardiovascular mortality due to depression in community-dwelling older adults. 
Table 1 Baseline characteristics according to depression score and physical activity group

\begin{tabular}{|c|c|c|c|c|c|c|c|c|}
\hline \multirow[b]{2}{*}{ Characteristic } & \multicolumn{3}{|c|}{ Depressive symptoms* } & \multicolumn{5}{|c|}{ Physical activity group score (lower score = less active) } \\
\hline & Low $(n=4600)$ & $\begin{array}{l}\text { High } \\
(\mathrm{n}=1252)\end{array}$ & p Value $\dagger$ & $\begin{array}{l}\text { I: } 0-3 \\
(n=653)\end{array}$ & $\begin{array}{l}\text { II: } 4-7 \\
(n=1914)\end{array}$ & $\begin{array}{l}\text { III: } 8-11 \\
(n=2546)\end{array}$ & $\begin{array}{l}\text { IV: } 12-15 \\
(n=739)\end{array}$ & p Value $\neq$ \\
\hline Age, years & $72.7 \pm 5.5$ & $73.2 \pm 5.9$ & 0.022 & $75.6 \pm 6.6$ & $73.5 \pm 5.8$ & $72.2 \pm 5.2$ & $70.9 \pm 4.2$ & $<0.001$ \\
\hline Race, \% white & 85.6 & 76.8 & $<0.001$ & 69.5 & 78.9 & 88.4 & 92.7 & $<0.001$ \\
\hline Education beyond $\mathrm{HS}, \%$ & 72.7 & 62.9 & $<0.001$ & 56.8 & 63.6 & 74.5 & 87.1 & $<0.001$ \\
\hline Current smoker, \% & 11.2 & 14.1 & 0.044 & 12.3 & 14.7 & 10.6 & 8.5 & 0.015 \\
\hline $\mathrm{BMI}, \mathrm{kg} / \mathrm{m}^{2}$ & $26.6 \pm 4.6$ & $27.0 \pm 5.2$ & 0.038 & $28.0 \pm 6.2$ & $27.3 \pm 5.0$ & $26.3 \pm 4.1$ & $25.5 \pm 3.7$ & $<0.001$ \\
\hline Systolic BP, mm Hg & $136.3 \pm 21.6$ & $137.4 \pm 22.5$ & 0.142 & $141.2 \pm 22.9$ & $137.8 \pm 22.0$ & $135.4 \pm 21.5$ & $133.1 \pm 20.6$ & $<0.001$ \\
\hline Diastolic BP, mm Hg & $70.8 \pm 11.3$ & $70.5 \pm 11.7$ & 0.398 & $71.6 \pm 12.1$ & $70.7 \pm 11.4$ & $70.3 \pm 11.1$ & $71.1 \pm 11.5$ & 0.233 \\
\hline Hypertension, \% & 42.8 & 50.4 & $<0.001$ & 55.2 & 49.2 & 41.1 & 34.0 & $<0.001$ \\
\hline CHD, \% & 18.7 & 25.8 & $<0.001$ & 28.2 & 21.5 & 17.9 & 17.6 & $<0.001$ \\
\hline Triglycerides, mg/dl & $138.6 \pm 76.1$ & $143.2 \pm 79.7$ & 0.073 & $149.4 \pm 91.3$ & $142.7 \pm 74.7$ & $137.0 \pm 76.6$ & $132.3 \pm 67.8$ & $<0.001$ \\
\hline CRP, mg/dl & $4.63 \pm 8.05$ & $5.65 \pm 9.84$ & $<0.001$ & $6.65 \pm 8.57$ & $5.60 \pm 9.94$ & $4.24 \pm 7.62$ & $3.40 \pm 6.34$ & $<0.001$ \\
\hline Cystatin C, mg/l & $1.11 \pm 0.33$ & $1.14 \pm 0.39$ & 0.042 & $1.26 \pm 0.50$ & $1.15 \pm 0.39$ & $1.09 \pm 0.28$ & $1.04 \pm 0.25$ & $<0.001$ \\
\hline Creatinine, mg/dl & $1.07 \pm 0.40$ & $1.06 \pm 0.40$ & 0.455 & $1.15 \pm 0.54$ & $1.08 \pm 0.44$ & $1.05 \pm 0.35$ & $1.04 \pm 0.25$ & $<0.001$ \\
\hline TCA, \% & 2.0 & 5.3 & $<0.001$ & 5.4 & 3.2 & 2.2 & 1.0 & $<0.001$ \\
\hline Non-TCA, \% & 0.6 & 2.3 & $<0.001$ & 1.8 & 1.1 & 0.9 & 0.3 & 0.003 \\
\hline
\end{tabular}

Data expressed as mean \pm SD for continuous variables or percentage for dichotomous variables.

*Depressive symptoms based on the Center for Epidemiological Studies-Depression Scale (10-item version): low (score $0-7)$ and high (score $\geq 8$ ).

$t p$ Values from two-sample $t$ tests for continuous and $\chi^{2}$ tests for dichotomous variables.

$\neq \mathrm{p}$ Values from linear regression for continuous and logistic regression for dichotomous variables.

$\mathrm{BP}$, blood pressure; BMI, body mass index; CHD, coronary heart disease; CHF, congestive heart failure; CRP, C reactive protein; HS, high school; HDL-C, high-density lipoprotein cholesterol; LDL-C, low-density lipoprotein cholesterol; TCA, tricyclic antidepressant use.

Other studies have reported that physical inactivity may be a partial mediator of the relationship between depression and cardiovascular events ${ }^{7}$ or mortality. ${ }^{18}$ In a group of initially medically stable patients with coronary artery disease, Brummett $e t$ al ${ }^{18}$ found that depressive symptoms were associated with increased mortality and with physical inactivity, as in the present study. These authors found that physical inactivity partially mediated the relationship between depression and mortality in patients with established coronary artery disease. A $13 \%$ reduction in the parameter estimate for Zung depression scores was reported after adding exercise to the Cox regression model used to identify factors predictive of survival in that population. Whooley et $a l^{7}$ reported that physical inactivity accounted for almost half of the association between depressive symptoms and cardiovascular events in a group of outpatients with stable coronary heart disease. These findings suggest that physical inactivity may play a somewhat more important role in a clinic-based population of individuals with established coronary heart disease than in community-dwelling older adults, although direct comparisons between studies is challenging because of differences in how physical activity is measured, in the methodology used to assess the contribution of physical inactivity to the increased risk of depression, and in the predictors of cardiovascular mortality accounted for in the analyses. There are also important differences in patient populations between studies which make direct comparisons difficult. Participants in the CHS were on average more than 20 years older than in the study by Brummett et al ${ }^{18}$ and $58 \%$ were women in the present study compared with only $18 \%$ in the studies by Brummett et al ${ }^{18}$ and by Whooley et al. ${ }^{7}$ Both studied

Table 2 Risk of cardiovascular mortality by depression score and physical activity

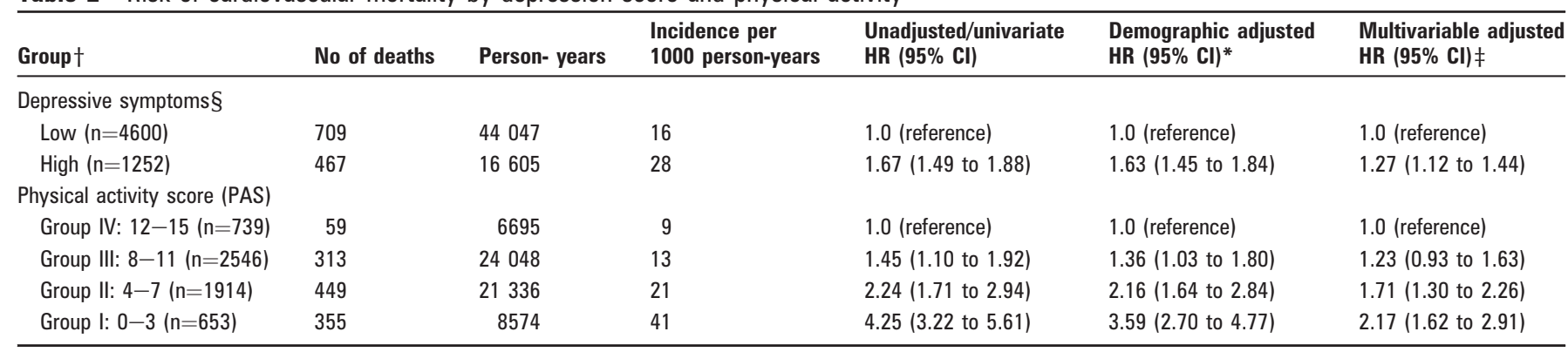

All $p$ values $<0.001$ except PAS group III: $p=0.008$ (unadjusted), $p=0.031$ (demographic) and $p=0.143$ (multivariable)

*Adjusted for age, race and gender.

†The number of participants in each group is taken from baseline.

$\ddagger$ Adjusted for age, race, gender, clinic location (four sites), education (less than high school, high school, beyond high school), body mass index (underweight, normal, overweight, obese), smoking (never, former, current), alcohol, high-density lipoprotein cholesterol, low-density lipoprotein cholesterol, diabetes, hypertension, coronary heart disease, congestive heart failure, stroke and antidepressant medication use.

$\S$ Depressive symptoms based on the Center for Epidemiological Studies-Depression Scale (10-item version): low (score 0-7) and high (score $\geq 8$ ) 
Table 3 Percentage reduction in the log hazard ratio of depressive symptoms for cardiovascular mortality after adding physical activity variables

\begin{tabular}{|c|c|c|c|c|c|c|c|}
\hline Variable & $\begin{array}{l}\text { Full cohort } \\
(\mathrm{N}=5852)\end{array}$ & $\begin{array}{l}\text { No CHD* } \\
(\mathrm{N}=4671)\end{array}$ & $\begin{array}{l}\text { CHD* } \\
(\mathrm{N}=1181)\end{array}$ & $\begin{array}{l}\text { Female } \\
(\mathrm{N}=\mathbf{3 3 7 4}) \\
\end{array}$ & $\begin{array}{l}\text { Male } \\
(\mathrm{N}=2478)\end{array}$ & $\begin{array}{l}\text { White } \\
(\mathrm{N}=4900)\end{array}$ & $\begin{array}{l}\text { Non-white } \\
\text { (N=952) }\end{array}$ \\
\hline Physical Activity Score (PAS) & 26 & 23 & 25 & 22 & 31 & 27 & 11 \\
\hline \multicolumn{8}{|l|}{ Components of PAS $†$} \\
\hline Exercise intensity & 10 & 11 & 8 & 13 & 23 & 11 & 4 \\
\hline Blocks walked & 15 & 13 & 16 & 11 & 37 & 15 & 15 \\
\hline Pace walked & 19 & 18 & 19 & 17 & 21 & 20 & 10 \\
\hline
\end{tabular}

Models were multivariable adjusted for age, race, gender, clinic location, education, body mass index, smoking, alcohol, high-density lipoprotein cholesterol, low-density lipoprotein cholesterol, diabetes, hypertension, coronary heart disease (CHD), congestive heart failure, stroke and antidepressant medication use.

All $p<0.05$ for $\log H R$ of depression except for results in non-white column ( $p$ range $0.16-0.24$ ).

*Population size with coronary heart disease shown is at study entry and changes over time as it was modelled as time-varying.

†Categorical variables.

‡Continuous variable.

patients with established cardiovascular disease, whereas only about $20 \%$ of participants in the CHS had established cardiovascular disease. Our findings should therefore be more generalisable to the older population and suggest that depressed individuals-regardless of whether they have established cardiovascular disease-may be able to reduce some of the risk due to depression by being more physically active. Of note, when the analysis was restricted only to CHS participants with established cardiovascular disease, our findings did not change substantially.

In the FINE study, ${ }^{19}$ as in the present study, depressive symptoms were associated with lower levels of self-reported physical activity, and both depressive symptoms and physical inactivity were associated with increased cardiovascular mortality. However, physical inactivity was felt to account for a smaller percentage of the increased mortality risk related to depression (ie, only $9 \%$ ), and the authors concluded that it was not likely to be a mediator of the relationship between depressive symptoms and increased mortality. ${ }^{29}$ There were important differences in the FINE study and the present report. The FINE population was smaller ( $\mathrm{n}=909$ vs 5888 in our study); the participants were from Finland, the Netherlands and Italy versus the USA; the participants were all men (vs $58 \%$ women in our study); only baseline measurements were used; a different measure of physical activity was used from that in the CHS; and

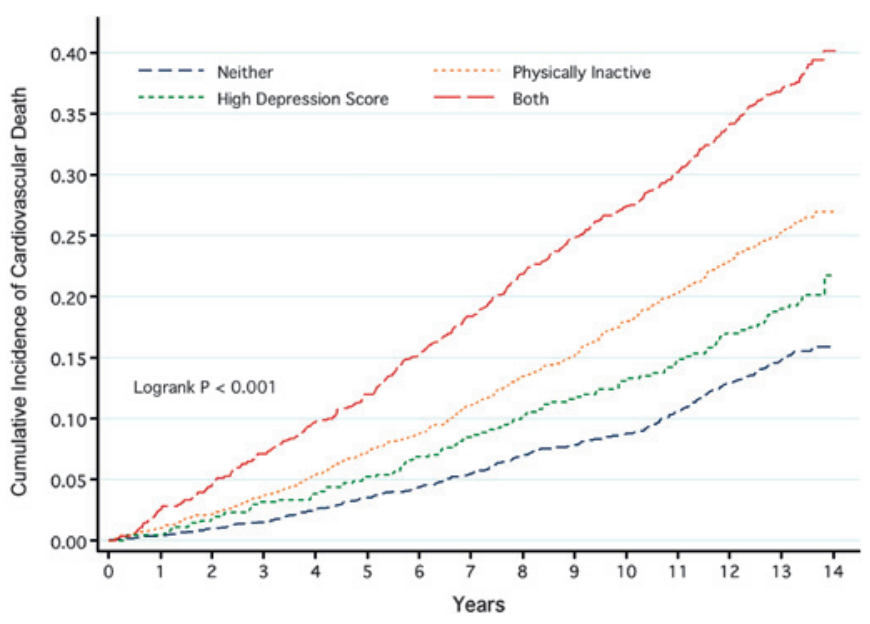

Figure 1 Cumulative incidence of cardiovascular death according to depression score and physical activity status. Number of cardiovascular deaths 1176/5852 (20.1\%). High depression score, Center for Epidemiological Studies Depression Score (CES-D) $\geq 8$; physical inactivity, Physical Activity Score (PAS) $\leq 7$. a different measure of depression was used (the Zung depression scale vs the CES-D in the CHS).

Not surprisingly, high depression scores and low physical activity were strongly associated in the present study. Individuals in the lowest physical activity group were $>3$ times as likely to have a high depression score as those in the most active group. Previous work in this area shows that the relationship between depression and physical inactivity is complex and bidirectional. Regular physical activity decreases the risk of depression ${ }^{29}$ whereas cessation of exercise can lead to the development of depressive symptoms. ${ }^{30}$ A recent systematic review provided evidence for the other direction of this relationship-namely, that baseline depression might lead to the development of a sedentary lifestyle or to a lower level of physical activity. ${ }^{9}$

The present study has several important strengths including the large number of participants, the inclusion of individuals with and without known coronary heart disease at baseline, and the relatively long period of follow-up (up to 14 years). The CHS also provides nearly $100 \%$ ascertainment of mortality and obtained many time-varying covariates with multiple repeated measures over time, reducing misclassification. In addition, the participants in the CHS were randomly selected from four geographically distinct communities and are likely to be more representative of the US population as a whole. The only previous study that examined whether physical inactivity mediates the relationship between depression and mortality among community-dwelling older adults without known heart disease included only men, ${ }^{19}$ whereas the present study included both men and women.

One limitation of our study is that the percentage reduction in the logHR of depression could represent not only mediation by physical inactivity but might also be due to confounding if physical inactivity preceded depressive symptoms. However, since depression has previously been shown to lead to inactivity, $^{8}$ much of this effect may be due to mediation. There are also limitations of the measures of both physical activity and depression. In the CHS, physical activity was determined by self-report rather than by objective measures and self-reported physical activity may be influenced by social desirability and social approval. ${ }^{31}$ Of note, however, studies have shown that self-report is highly correlated to more objective measures of physical activity and fitness, including treadmill performance ${ }^{32}$ and maximal oxygen consumption. ${ }^{33}$ Since physical activity has many dimensions, a strength of our study is that participants were asked about a variety of different domains of physical activit, including multiple common leisure time activities. In addition, information was obtained about a variety of different attributes of these activities including frequency, duration, pace and intensity. 
Another limitation of our study is that depressive symptoms were assessed using the CES-D rather than by a structured interview. The CES-D is a valid and reliable instrument for assessing depressive symptoms in large community samples including community-dwelling older adults. ${ }^{34}$ The CES-D does not result in a clinical diagnosis of a major depressive disorder (MDD) but its construct and predictive validity have been established in older adults in general ${ }^{25}$ and in the CHS cohort in particular. ${ }^{4}$ The prevalence of significant depressive symptoms of $20-30 \%$ in this cohort (ie, participants with CES-D scores above the cut-off) is higher than the approximately $5 \%$ prevalence of $\mathrm{MDD}$ in community-dwelling older adults, ${ }^{35}$ which indicates that most of the participants classified as 'depressed' based on the CES-D had sub-threshold depressive symptoms. Additional studies are needed to examine the role of physical inactivity as a mediator of the adverse health risks among clinically depressed individuals.

In summary, the present study shows that physical inactivity accounts for a significant proportion (approximately 25\%) of the increased cardiovascular mortality risk due to depressive symptoms in adults aged $\geq 65$ years. These data suggest that preventive health and wellness programmes in older adults, particularly those with depression, should focus on encouraging enrolment and continued participation in exercise programmes. Future research might examine whether incentives could be used to change health behaviour in certain individuals, as has been done in other settings. ${ }^{36} 37$ Positive financial incentives, health insurance rebates, transportation vouchers or health club memberships might enhance participation of older adults with depression in these programmes and thereby reduce healthcare utilisation and the risk of cardiovascular events.

Funding The research reported in this article was supported by contract numbers N01-HC-85239, N01-HC-85079 through N01-HC-85086, N01-HC-35129, N01 HC-15103, N01 HC-55222, N01-HC-75150, N01-HC-45133, grant number U01 HL080295 from the National Heart, Lung, and Blood Institute, with additional contribution from the National Institute of Neurological Disorders and Stroke. A full list of principal CHS investigators and institutions can be found at http://www.chs-nhlbi. org/pi.htm. RCZ was supported by grant R24AT004641 from the National Center for Complementary and Alternative Medicine and by the Miller Family Scholar Program of the Johns Hopkins Center for Innovative Medicine.

\section{Competing interests None.}

Ethics approval The institutional review board at each centre approved the study and participants gave informed consent.

Contributors All authors had full access to all of the data in the study and take responsibility for the integrity of the data and the accuracy of the data analysis.

Provenance and peer review Not commissioned; externally peer reviewed.

\section{REFERENCES}

1. Mehta KM, Yaffe K, Langa KM, et al. Additive effects of cognitive function and depressive symptoms on mortality in elderly community-living adults. J Gerontol $A$ Biol Sci Med Sci 2003;58:M461-7.

2. Penninx BW, Guralnik JM, Mendes de Leon CF, et al. Cardiovascular events and mortality in newly and chronically depressed persons $>70$ years of age. Am J Cardiol 1998;81:988-94

3. Rovner BW, German PS, Brant LJ, et al. Depression and mortality in nursing homes. JAMA 1991;265:993-6.

4. Schulz R, Beach SR, Ives DG, et al. Association between depression and mortality in older adults: the Cardiovascular Health Study. Arch Intern Med 2000;160:1761-8.

5. Callahan CM, Wolinsky FD, Stump TE, et al. Mortality, symptoms, and functional impairment in late-life depression. J Gen Intern Med 1998;13:746-52.

6. Shah A. Can depression and depressive symptoms predict mortality at 18-month follow-up in acutely medically ill inpatients over the age of 80 years? Int J Geriatr Psychiatry 1998;13:240-3.
7. Whooley MA, de Jonge P, Vittinghoff E, et al. Depressive symptoms, health behaviors, and risk of cardiovascular events in patients with coronary heart disease. JAMA 2008;300:2379-88.

8. Bonnet F, Irving K, Terra JL, et al. Anxiety and depression are associated with unhealthy lifestyle in patients at risk of cardiovascular disease. Atherosclerosis 2005:178:339-44.

9. Roshanaei-Moghaddam B, Katon WJ, Russo J. The longitudinal effects of depression on physical activity. Gen Hosp Psychiatry 2009;31:306-15.

10. Bijnen FC, Caspersen CJ, Feskens EJ, et al. Physical activity and 10-year mortality from cardiovascular diseases and all causes: the Zutphen Elderly Study. Arch Intern Med 1998;158:1499-505.

11. Bijnen FC, Feskens EJ, Caspersen CJ, et al. Baseline and previous physical activity in relation to mortality in elderly men: the Zutphen Elderly Study. Am J Epidemiol 1999:150:1289-96.

12. Fried LP, Kronmal RA, Newman AB, et al. Risk factors for 5-year mortality in olde adults: the Cardiovascular Health Study. JAMA 1998;279:585-92.

13. Hakim AA, Petrovitch $\mathrm{H}$, Burchfiel CM, et al. Effects of walking on mortality among nonsmoking retired men. N Engl J Med 1998;338:94-9.

14. Lee IM, Hsieh CC, Paffenbarger RS. Exercise intensity and longevity in men. The Harvard Alumni Health Study. JAMA 1995:273:1179-84.

15. Leon AS, Connett J, Jacobs DR, et al. Leisure-time physical activity levels and risk of coronary heart disease and death. The Multiple Risk Factor Intervention Trial. JAMA 1987:258:2388-95.

16. Manini TM, Everhart JE, Patel KV, et al. Daily activity energy expenditure and mortality among older adults. JAMA 2006;296:171-9.

17. Blumenthal JA, Babyak MA, Carney RM, et al. Exercise, depression, and mortality after myocardial infarction in the ENRICHD trial. Med Sci Sports Exerc 2004:36:746-55

18. Brummett BH, Babyak MA, Siegler IC, et al. Effect of smoking and sedentary behavior on the association between depressive symptoms and mortality from coronary heart disease. Am J Cardiol 2003:92:529-32.

19. Kamphuis $\mathbf{M H}$, Geerlings Ml, Tijhuis MAR, et al. Physical inactivity, depression, and risk of cardiovascular mortality. Med Sci Sports Exerc 2007;39:1693-9.

20. Fried LP, Borhani NO, Enright P, et al. The Cardiovascular Health Study: design and rationale. Ann Epidemiol 1991;1:263-76.

21. Ives DG, Fitzpatrick AL, Bild DE, et al. Surveillance and ascertainment of cardiovascula events. The Cardiovascular Health Study. Ann Epidemiol 1995:5:278-85.

22. Taylor HL, Jacobs DR, Schucker B, et al. A questionnaire for the assessment of leisure time physical activities. J Chronic Dis 1978:31:741-55.

23. Folsom AR, Jacobs DR Jr, Caspersen CJ, et al. Test-retest reliability of the Minnesota Leisure Time Physical Activity Questionnaire. J Chronic Dis 1986;39:505-11.

24. Siscovick DS, Fried L, Mittelmark M, et al. Exercise intensity and subclinical cardiovascular disease in the elderly. The Cardiovascular Health Study. Am J Epidemiol 1997;145:977-86.

25. Andresen EM, Malmgren JA, Carter WB, et al. Screening for depression in well older adults: evaluation of a short form of the CES-D (Center for Epidemiologic Studies Depression Scale). Am J Prev Med 1994;10:77-84.

26. Radloff LS. The CES-D Scale: a self-report depression scale for research in the general population. Appl Psychol Meas 1977;1:385-401.

27. Ariyo AA, Haan M, Tangen CM, et al. Depressive symptoms and risks of coronary heart disease and mortality in elderly Americans. Cardiovascular Health Study Collaborative Research Group. Circulation 2000;102:1773-9.

28. Newman AB, Sachs MC, Arnold AM, et al. Total and cause-specific mortality in the cardiovascular health study. J Gerontol A Biol Sci Med Sci 2009:64:1251-61.

29. Teychenne M, Ball K, Salmon J. Physical activity and likelihood of depression in adults: a review. Prev Med 2008;46:397-411.

30. Berlin AA, Kop WJ, Deuster PA. Depressive mood symptoms and fatigue after exercise withdrawal: the potential role of decreased fitness. Psychosom Med 2006;68:224-30

31. Adams SA, Matthews CE, Ebbeling CB, et al. The effect of social desirability and social approval on self-reports of physical activity. Am J Epidemiol 2005;161:389-98.

32. Bowles HR, FitzGerald SJ, Morrow JR, et al. Construct validity of self-reported historical physical activity. Am J Epidemiol 2004;160:279-86.

33. Kurtze N, Rangul V, Hustvedt BE, et al. Reliability and validity of self-reported physical activity in the Nord-Trøndelag Health Study - HUNT 1. Scand J Public Health 2008:36:52-61.

34. Beekman A, Deeg D, Van Limbeek J, et al. Criterion validity of the Center for Epidemiologic Studies Depression scale (CES-D): results from a community-based sample of older subjects in the Netherlands. Psychol Med 1997;27:231-5

35. Gum AM, King-Kallimanis B, Kohn R. Prevalence of mood, anxiety, and substanceabuse disorders for older Americans in the national comorbidity survey-replication. Am J Geriatr Psychiatry 2009;17:769-81.

36. Marteau TM, Ashcroft RE, Oliver A. Using financial incentives to achieve healthy behaviour. BMJ 2009:338:b1415.

37. Sutherland K, Christianson JB, Leatherman S. Impact of targeted financial incentives on personal health behavior: a review of the literature. Med Care Res Rev 2008:65:36-78S 